\title{
20 Post-primary Pulmonary Tuberculosis
}

\author{
M. Monir Madkour, Y. Abusabaah, Ali Ben Mousa, Ali Al Masoud
}

\section{CONTENTS}

20.1 Pathogenesis and Pathology 313

20.2 Clinical Features and Complications 315

20.2.1 Hemoptysis in Pulmonary Tuberculosis 316

20.2.2 Massive Hemoptysis in Tuberculosis 316

20.2.3 Rasmussen Aneurysm 317

20.2.4 Endobronchial Tuberculosis 318

20.2.5 Bronchiectasis as a Complication of Post-primary Pulmonary TB 318

20.2.6 Pneumothorax as Complication of Post-primary Pulmonary TB 318

20.2.7 ARDS and Tuberculosis 319

20.2.8 Tuberculous Bronchopleural Fistula 319

20.2.9 Aspergilloma (Mycetoma) 319

20.2.10 Tuberculomas 320

20.2.11 Tuberculous Pulmonary Gangrene 320

20.2.12 Tuberculosis, Lung Cancer and Other Neoplasia 320

20.3 Diagnosis of Post-primary Pulmonary TB 320

20.3.1 Imaging Features of Post-primary Pulmonary TB 320

20.3.2 Microbiology of the Sputum and Bronchial Aspirate 321

20.3.3 Bronchoscopic Diagnosis 321

20.3.4 Tuberculin Skin Test 322

20.3.5 Other Diagnostic Investigations 322

20.4 Treatment of Post-primary Pulmonary TB 322

20.4.1 Response to Treatment 324

References 324
Post-primary pulmonary tuberculosis is a chronic disease commonly caused by either endogenous reactivation of a latent infection or exogenous re-infection by Mycobacterium tuberculosis. It has other synonyms derived mostly from the route of transmission of the infection or from the age of the patient at the onset of the disease, including endogenous reactivation primary tuberculosis, exogenous re-infection pulmonary tuberculosis, or adult-onset pulmonary tuberculosis. The term being used here is 'post-primary pulmonary tuberculosis' to include both re-infection and reactivation forms. The clinical features of the disease are not specific, and the imaging features are suggestive but can simulate other diseases. The definitive diagnosis depends on the identification of $M$. tuberculosis bacilli, using conventional microbiological methods of sputum smear and culture or radiometric culture methods such as BACTEC or DNA probe PCR-based assays which can identify drug-resistant strains as well. Sputum smear and culture remain the most important investigative methods. Smear-negative sputum may delay the diagnosis for 4-8 weeks or longer if the culture is also negative. A presumptive diagnosis based on the clinical and radiographic features should be made with initiation of treatment after the exclusion of other possible causes of the radiographic findings.

Infection with HIV is a great risk for the development of either endogenous reactivation or exogenous re-infection pulmonary disease (Millar and Horne 1979; Barnes et al. 1991; Heyderman et al. 1998). The development of drug-resistant pulmonary tuberculosis is a real global concern that indicates a failure of the tuberculosis control program. The current treatment is both toxic and expensive, and new drug development is sparse at present.

\section{1 \\ Pathogenesis and Pathology}

The development of post-primary pulmonary tuberculosis as a result of endogenous reactivation or exog- 
enous re-infection in low-risk and high-risk areas has been debated for many years among authors (Glynn et al.2001). Balasubramanian and colleagues reviewed the world literature on these issues and added their own views (Balasubramanian et al. 1994). Schools of thought adopted by those researchers based on microbiological studies of tissue specimens from the lung and lymph nodes of the primary complex. Some authors quoted by reviewers suggested that the primary complex is sterile within 5 years, while other authors suggest that virulent bacilli lie dormant in a metastatic site seeded hematogenously within the vulnerable region. Cultures of apical lung lesions yielded viable bacilli in $25 \%-76 \%$ as reported by some of the quoted authors, and transmission via the bloodstream was suggested.

Other researchers examined the sputum and urine cultures in patients living in Bangalore, India, and compared the bacilli virulence, INH sensitivity, and phage typing of the isolates and drew conclusions about the biological evidence of exogenous re-infection in that study. Epidemiologists have noted that high- or lowrisk incidence rates of infection in different areas of the world played an important role (in exogenous reinfection or endogenous reactivation). In areas with a risk greater than $1 \%$ of developing countries, with the likelihood of repeated episodes of droplet infection via the airway, the disease is more likely to be due to exogenous re-infection. In areas of low-risk (less than $0.05 \%$ ), as in developed countries, endogenous reactivation is more likely to be the cause of the disease. Some other authors gave evidence of the contributions of both exogenous re-infection as well as endogenous reactivation. Balasubramanian et al. hypothesized that the implantation of the vulnerable region is directly transmitted via the airway (exogenous re-infection). They also concluded that more studies using genetic fingerprinting of Mycobacterium bacilli isolates would help in the future in disease control in endemic areas and in the development of new vaccines. Immune suppression resulting from HIV infection leads to higher rates of co-infection with tuberculosis.

Recent molecular epidemiological studies have indicated that up to $40 \%$ of newly diagnosed tuberculous patients and over $70 \%$ of recurrences may be due to exogenous re-infection (Stead and Bates 2000; Bates et al. 2001). Among the immunosuppressed patients due to HIV, exogenous re-infection was the leading mechanism. Endogenous reactivation contributed to only $16 \%$ of patients in a low-risk area in developed countries and more than $70 \%$ in a high-risk area in developing countries (Alland et al. 1994; Brande et al. 1998; Small et al. 1994; van Rie et al. 1999; McDonough et al. 2000; Rook and Zumla 2001; Caminero et al. 2001; Bandera et al. 2001; Wallis and Johnson 2001).

Caminero and colleagues (2001) reported 912 patients with culture-proved pulmonary tuberculosis between 1991 and 1996 on a Spanish island with a moderate risk rate of incidence. They were treated and followed up for at least 12 months after completing chemotherapy and were culture-negative. Twentythree patients $(2.5 \%)$ became culture positive again. DNA fingerprinting results were available from 18 patients with recurrence of the disease. DNA fingerprinting was available of pretreatment and recurrent isolates and was reviewed. In 8 patients (44\%), the genotype of the recurrent isolate showed a different pattern to the pretreatment isolate. These authors concluded that in 8 patients, 2 of them HIV-positive, exogenous re-infection was the cause of the recurrent pulmonary tuberculosis. Immune suppression or reduction of immune responses may also occur in certain diseases such as silicosis, diabetes mellitus, or with corticosteroid and other immunosuppressive drugs used for malignancies or connective tissue diseases.

Regardless of the mode of infection (exogenous or endogenous), the apex of the lung (apical-posterior segments) is the most common site of post-primary pulmonary tuberculosis. Specific factors permitting the progression of tuberculosis in most cases are not yet known (Wallis and Johnson 2001).

The host immune response and the role of the cellmediated immunity of activated macrophages and T-cells and the expression of cytokines (Garcia et al. 2002) in response to M. tuberculosis glycolipids and lipoproteins have been discussed in the pathogenesis of primary pulmonary tuberculosis in Chap. 17. Post-primary pulmonary tuberculosis commonly affects the apical and posterior segments of the upper lobe or the superior segment of a lower lobe. After its localization, inflammatory granulomatous nodular formations with cellular infiltrates, fibrosis, central necrosis, and caseation may take place. Hilar and paratracheal lymphadenitis (Woodring 1986) in post-primary tuberculosis is a rare occurrence and reported in approximately $5 \%$ of patients (see Figs. 23.18, 23.20, 23.21, 23.22, 23.39, 23.40b). Healing with fibrosis and calcification (Fig. z.25) of lung parenchymal lesion may occur, leading to traction of the trachea (Fig. 23.21, 23.22, 23.23), and in late advanced stages when the lung is destroyed and replaced by extensive fibrosis, displacement of the mediastinum may occur (Fig. 23.24).

The initial upper lobe infiltration may form a pneumonic consolidation, and cavitation often 
occurs in $40 \%-80 \%$ of patients (Fig. 23.20, 23.26a, b) (Rohenberg and Shaw 1996). Tuberculous consolidation with cavity formation may expand and form a lung abscess discharging large numbers of the bacilli in the sputum as was noted in one of our patients (Fig. 23.26d). Tuberculous cavities with areas of necrosis and caseation may rupture into the pleura, leading to empyema (Fig. 23.34b) or a bronchopleural fistula (Fig. 23.36). Rupture of a tuberculous cavity into the trachea or bronchi is a common complication and occurs in up to $40 \%$ of patients (Rohenberg and Shaw 1996). Bronchogenic spread of necrotic and caseous tissue loaded with the bacilli to other parts of the lung fields on the same side or opposite lung may occur (Figs. 23.19, 23.20, 23.27b,c, 23.28b,c). Infection starts at the newly seeded sites with nodular infiltrate bronchopneumonia or similar tissue destruction and fibrosis to the lung parenchyma (Figs. 23.25, 23.26a, z.27b, c, 23.28b). A severe form of transbronchial spread of infection takes the form of dissemination in both lungs (Fig. 23.28c).

Endobronchial involvement of the bronchial wall is common, leading to scarring and luminal narrowing and post-stenotic emphysema (Figs. 23.22b, 23.30a, b, $23.25,23.31 \mathrm{~b}$ ), atelectasis, and cystic or tubular bronchiectasis due to traction or endobronchial fibrosis as a common complication of the tuberculous disease process. It is often located in the upper lobes but can occur at any other site (Figs. 23.30a, b, 23.31a, b). Posttuberculous bronchiectasis, although often asymptomatic, may cause hemoptysis in these patients.

A tuberculous cavity larger than $25 \mathrm{~mm}$ in diameter may persist long after successful antibiotic treatment. Colonization with fungi forming a ball of mycetoma may rarely occur (Fig. 23.26b, 23.37a,b), and hemoptysis may rarely be a presenting complaint.

Tuberculous granulomatous tissue nodules may be encapsulated with connective tissue during variable stages of disease healing and activity, leading to tuberculomas found in $3 \%-6 \%$ of patients. It may be single or multiple with a central area of necrosis or calcification (Figs. 23.32a-d). Tuberculomas may occur in both primary and post-primary pulmonary tuberculosis.

In bovine tuberculosis, the $M$. bovis bacilli are bacteriologically distinctive from $M$. tuberculosis but otherwise cause identical pathogenesis, lung lesions, and clinical disease. In humans, M. bovis is a zoonotic disease that has been virtually eliminated in developed countries and other developing countries that practice pasteurization of milk and immunization of dairy herds (Grange et al. 1994; Dannenberg 2001). The organism is excreted in the milk from cows, goats, and sheep with udder tuberculosis. It can also be transmitted to humans from cows as an airborne infection (Geppert and Leff 1979; Liesegang and Cameron 1980; Sauret et al. 1992). The incidence of $M$. bovis in humans is higher in rural areas with infected herds (Moda et al. 1996). Ingestion of contaminated milk, particularly by young children living in developing countries, leads to establishment in the cervical and less frequently in the axillary lymph nodes (scrofula). The bacilli may also affect the intestine, kidney, bones, and central nervous system in endemic areas with a similar pathogenicity to M. tuberculosis (Moda et al. 1996). HIV patients co-infected with $M$. bovis have been reported in San Diego, USA, near the border with Mexico (Dankner et al. 1993). Nontuberculous mycobacterial organisms may also cause a similar pulmonary infection (see Chap. 11).

\section{2 \\ Clinical Features and Complications}

Post-primary tuberculosis often presents with a gradual onset of symptoms that may be tolerated by the patient. The duration of symptoms before presentation may vary widely, from 3 days to 23 months (Dahmash et al. 1995; Maartens and Beyers 2002). The presenting features may initially be related to the respiratory system or present as constitutional symptoms or both. Cough is the most frequently reported presenting feature. Initially, it is dry but later becomes productive. The sputum may be mucoid, muco-purulent, blood-stained, or with massive hemoptysis. Chest pain due to associated pleurisy or pneumothorax may be a presenting symptom. Dyspnea due to tuberculous pneumonia or bilateral fibrocavitary disease may be the presenting symptom. Fever with sweating and chills are common, particularly at night. Other constitutional symptoms including weakness, anorexia, and weight loss, which are nonspecific, may also be present (Dunlap et al. 2000; Johnson and Ellner 2000).

Symptoms related to extrapulmonary tuberculosis such as tuberculous monoarthritis, Pott's disease, genitourinary symptoms, or other organ involvement might accompany the respiratory symptoms at the time of presentation. We retrospectively reviewed the records of 176 adult patients with post-primary pulmonary tuberculosis who had a positive sputum culture for M. tuberculosis. They attended our hospital between 1998 and 2000. The frequency of history/ symptoms, physical findings, and imaging features of these patients are presented in Table 20.1. 
Table 20.1. Clinical features of 176 patients with culture-positive post-primary pulmonary tuberculosis

\begin{tabular}{lrl}
\hline Clinical features & No. of patients & Percentage \\
\hline Symptoms: & & \\
Cough & 166 & 94.3 \\
Expectoration & 128 & 72.7 \\
Hemoptysis & 40 & 23.0 \\
Fever & 135 & 76.7 \\
Sweating & 80 & 45.4 \\
Weight loss & 98 & 55.7 \\
Dyspnea & 76 & 43.2 \\
Chest pain & 58 & 32.9 \\
Signs: & & \\
Crackles & 105 & 59.6 \\
Localized wheezes & 23 & 13.0 \\
Bronchial sound & 55 & 31.2 \\
Diminished sound & 66 & 37.5 \\
Clubbing & 20 & 11.4 \\
Extrapulmonary & 16 & 9.0 \\
Bronchoscopy & 28 & 16.0 \\
\hline
\end{tabular}

The frequency of these symptoms may vary, being more intense among those co-infected with HIV (Corbett et al.2000).

The frequency of symptoms was also reported from a large tuberculosis center in Riyadh for 1566 hospitalized patients with pulmonary tuberculosis during 1983-1987. Fever and constitutional symptoms were reported in $77.7 \%$, cough with or without expectoration in $94.3 \%$, and hemoptysis in $40.7 \%$ (AlHajjaj et al. 1991). In another series from the south of Saudi Arabia, Al Wabel et al. (1995) reported on 190 patients with post-primary pulmonary tuberculosis who were hospitalized over a 2.5 -year period. Cough was noted in $84 \%$, expectoration in $65 \%$, hemoptysis in $23 \%$, fever and constitutional symptoms in over $40 \%$ of patients. Pulmonary tuberculosis in elderly patients with underlying and concomitant other chronic illnesses in 80 patients were reported from Riyadh (Dahmash et al. 1995). These patients had diabetes mellitus or malignancies, and some were on steroids, and these illnesses occurred in $86 \%$ of them, but none had HIV. Cough was noted in $85 \%$, expectoration in $60 \%$, fever in $66 \%$, hemoptysis in $17.5 \%$, anorexia, weight loss, and other constitutional symptoms in over $50 \%$. Choyke and colleagues (1983) reported on 103 patients with adult-onset pulmonary tuberculosis: $85 \%$ were symptomatic, with fever in $40 \%$, cough in $37 \%$, weight loss in $23.6 \%$, and hemoptysis in $8 \%$.

Physical examination of the chest may be normal despite the presence of pulmonary infiltrations depicted by chest radiography. Displacement of the trachea due to lung fibrosis and collapse may be found (Fig. 23.21). Chest wall retraction due to fibrosis or prominence due to associated pleural effusion may be found. Features of consolidations with crepitation and bronchial breathing may be detected. Localized wheezes may be present, indicating endobronchial disease. Other systemic features including pallor, rarely clubbing of the fingers and toes in chronic cases, weight loss, dyspnea, and other extrapulmonary involvement may be found on physical examination.

Patients with post-primary pulmonary tuberculosis may present with clinical features similar to those of community-acquired pneumonia. Al-Zeer and colleagues (1998) reported a series of 64 patients admitted to hospital during the pilgrimage season to Mecca in 1994 with an initial diagnosis of community-acquired pneumonia. All patients came from developing countries. Microbiologically proven $M$. tuberculosis was found in 13 patients $(20.3 \%)$ and was the most common cause of pneumonia among this group.

\subsection{1 \\ Hemoptysis in Pulmonary Tuberculosis}

Hemoptysis is often an alarming presenting symptom in patients with tuberculosis. It may vary in severity from a slight tinge of blood mixed with the sputum, to mild, moderate, or severe, massive, lifethreatening hemoptysis.

In our series of 176 patients with microbiologically proved pulmonary tuberculosis, 28 patients $(16 \%)$ had slight to mild hemoptysis that was managed with chemotherapy and conservative treatment. Active pulmonary tuberculosis with or without cavitation or post-tuberculous bronchiectasis is the most common cause among these patients. Rarely, the development of bronchial carcinoma in these patients as well or mycetoma or other unrelated illnesses could be the cause of hemoptysis in tuberculous patients (Stebbings and Lim 1999; Hirshberg et al. 1997).

\subsection{2 \\ Massive Hemoptysis in Tuberculosis}

Life-threatening massive hemoptysis due to active tuberculosis or post-tuberculous bronchiectasis has been reported as 'common in comparison to other causes listed in many publications', as reported by Hsiao et al. (2001) from the USA. Life-threatening, massive hemoptysis is defined by most authors as 
expectoration of at least $200 \mathrm{ml}$ of blood in $24 \mathrm{~h}$, significant drop of hemoglobin requiring blood transfusion, or failure to respond to conservative treatment such as oxygen supplement, morphine, and antibiotics (Wong et al. 2002; Abal et al. 2001; Lee et al. 2000; Hsiao et al. 2001; Conlan et al. 1983). In South Africa, Conlan et al. (1983) reviewed 123 patients with massive hemoptysis due to different causes. Tuberculosis was by far the most common cause, either due to culture-proved active disease or bronchiectasis as a sequela to tuberculosis. These authors reported that active pulmonary tuberculosis as a cause of massive hemoptysis was found in 47 patients (38\%), 24 men and 23 women aged between 19 and 60 years. Thirty-seven patients had bronchiectasis, and 17 (45.9\%) had bilateral upper lobe bronchiectasis secondary to former tuberculosis. The total number of patients with massive hemoptysis with or who had tuberculosis was 64 (52\%). The remaining causes were chronic narcotizing pneumonitis in 11, lung abscess in 6 , lung cancer in 6 , primary fungal pneumonia in 4 , bronchovascular fistula in 5 , and miscellaneous causes in 7 patients.

In France, Mal and colleagues (1999) reported the intermediate and long-term outcome of bronchial artery embolization (BAE) performed on 46 patients with massive hemoptysis. Tuberculosis (active or sequela) was found in 23 patients (50\%) as a cause of the massive hemoptysis. Idiopathic in 10, bronchiectasis in 4, lung cancer in 2, and 1 patient each due to other various causes.

The outcome of BAE was favorable with an immediately successful result in stopping the bleeding, but complications were also frequent. Revascularization may occur with recurrence of hemoptysis, technical failure in BAE, spinal cord injury related to invisible anastomotic connections between the bronchial circulation and the anterior spinal artery. The authors recommended that BAE should be avoided in patients with minor hemoptysis.

In the USA, Hsiao et al. (2001) from Stanford reported the assessment of modalities of investigations used for the localization of the site of bleeding in patients with massive hemoptysis. They reviewed the records of 28 patients seen between 1988 and 2000 who presented with massive hemoptysis. They found 16 patients with tuberculosis (57\%), 14 with tuberculous bronchiectasis, and 2 with active disease, bronchogenic carcinoma in 2, and other causes had 1 patient each. These authors noted, 'Contrary to the statistics reported in many recent series, tuberculous bronchiectasis is the most common underlying etiology for massive hemoptysis in our patients'.
The authors also indicated that the overall mortality of patients with massive hemoptysis was $7 \%-80 \%$ and operative mortality was $30 \%-40 \%$ as they found from reviewing the literature on the subject. These authors reported, 'The angiographic signs in hemoptysis include hyperplasia of the bronchial artery trunk and branches, bronchopulmonary anastomoses, and bronchial arterial aneurysms', which were seen on angiography of these patients. They concluded that fibroptic bronchoscopy before BAE is unnecessary in patients with hemoptysis of known origin.

In Kuwait, Abal et al. (2001) prospectively studied 52 hospitalized patients with hemoptysis of variable degrees of severity over a period of 1 year. Twenty had blood-stained sputum, and 32 had frank hemoptysis including 16 with massive blood expectoration. They found that pulmonary tuberculosis (active or old) was the most common cause, found in 17 patients (32.7\%). Other causes included carcinoma in 5, bronchitis in 3, 1 patient each due to other causes, and unknown cause in 13 patients. These authors managed $80.8 \%$ of their patients conservatively, and only $19 \%$ required BAE or surgery. Recurrent hemoptysis occurred in $12 \%$ at the 1-year follow-up in this series.

In a retrospective study of BAE for massive hemoptysis, Wong et al. (2002) from South Africa reported on 165 patients, and bilateral post-tuberculous bronchiectasis was the most common cause (75\%). The short-term outcome of BAE was satisfactory in controlling hemoptysis in all patients. Thoracic aortography was done during the initial assessment, and they noted pathologic enlargement of the bronchial arteries and the presence of nonbronchial systemic collaterals with arteriovenous shunting including intercostal arteries. One patient had transient paraparesis, and the authors noted that the presence of spinal arteries was not considered a contraindication to embolization.

\subsection{3}

\section{Rasmussen Aneurysm}

This is a rare phenomenon that can cause massive, life-threatening hemoptysis. It involves invasion of a peripheral pulmonary artery located within a tuberculous cavity often in the upper lobe. Invasion of the arterial wall by tuberculous granulation tissue leads to granulomatous vasculitis with replacement of the adventitia and media with fibrin during the process of healing. This will lead to weakening of the arterial wall with mycotic pseudoaneurysmal formation in one or more locations (see Chapter 43). Rupture of 
the aneurysm will lead to massive, life-threatening hemoptysis as reported in 5\% of postmortem cases (Winer-Muram and Rubin 1990; Kim et al. 2001).

\subsection{4}

\section{Endobronchial Tuberculosis}

Endobronchial tuberculosis is a common complication of post-primary pulmonary tuberculosis. It may occur in up to $40 \%$ of patients, and the most common source of bronchial wall infection is a contiguous tuberculous cavity (Rohenberg and Shaw 1996). Rarely, the bronchial wall may be affected by a hilar or paratracheal tuberculous caseating lymphadenitis. Hematogenous or lymphatic spread of infection to the bronchial wall may occur (Buckner and Walker 1990). The role of the bronchial tree in the spread of tuberculosis to other parenchymal segments or lobes on the same or opposite side by transbronchial spread of caseous material leading to bronchopneumonia is well recognized (Im et al. 1993). Hoarseness of voice due to laryngeal involvement and localized wheezes due to bronchial disease in the chest are common clinical features. The imaging features of plain radiography and CT may show multiple nodular opacities, bronchial wall thickening, post-stenotic dilatation, lobar hyperinflation, pulmonary collapse, and atelectasis (Figs. 23.27 and 23.28).

Endobronchial tuberculosis, its definition, epidemiology, pathogenesis and pathology, classification, clinical features, diagnosis, the role of bronchoscopy in the diagnosis and management, and the treatment are considered in a separate chapter (see Chap. 21).

\subsection{5 \\ Bronchiectasis as a Complication of Post-primary Pulmonary TB}

Bronchiectasis is a common sequela of pulmonary tuberculosis (primary and post-primary). It may occur primarily as a result of endobronchial tuberculosis with irreversible bronchial wall dilatation (Lee et al. 1991). Secondary tuberculous bronchiectasis occurs as a result of lung parenchymal destruction with fibrosis (traction bronchiectasis). Based on high-resolution $\mathrm{CT}$, bronchiectasis was found in $27 \%$ of patients with pulmonary tuberculosis, and the upper lobes were the most common site (Cartier et al. 1999).

In our series of 176 patients with pulmonary tuberculosis, 17 (9.6\%, see Table 20.2) had imaging features of tuberculous bronchiectasis (Figs. 23.9, $23.27 c, 23.28 c, 23.29,23.30$, and 23.31). Typically, the
Table 20.2. Imaging features of 176 patients with culture-positive post-primary pulmonary tuberculosis

\begin{tabular}{|c|c|c|}
\hline Imaging features & No. of patients & Percentage \\
\hline Unilateral-parenchymal & 119 & 67.6 \\
\hline Bilateral-parenchymal & 57 & 32.3 \\
\hline Infiltrates and consolidation & 144 & 81.8 \\
\hline Cavities & 61 & 34.6 \\
\hline Bronchiectasis & 17 & 9.6 \\
\hline Destroyed lungs & 2 & 1.1 \\
\hline $\begin{array}{l}\text { Calcifications } \\
\text { (LN, parenchymal and pleural) }\end{array}$ & 22 & 12.5 \\
\hline Pneumothorax & 8 & 4.5 \\
\hline $\begin{array}{l}\text { Lymphadenopathy } \\
\text { (hilar and paratracheal) }\end{array}$ & 19 & 10.8 \\
\hline $\begin{array}{l}\text { Lung infiltrates with pleural } \\
\text { effusion }\end{array}$ & 46 & 26.1 \\
\hline Mycetoma & 2 & 1.1 \\
\hline Tuberculomas & 3 & 1.7 \\
\hline Bronchopleural fistula & 1 & 0.5 \\
\hline
\end{tabular}

apical and posterior segments of the upper lobes are the most common sites of tuberculous bronchiectasis (Fig. 23.27c). Chest radiography may show ring shadows with occasional fluid levels (Figs. 23.9 and 23.29). Features on CT and HRCT will show the details of the bronchial wall changes (Figs. 23.28c, 23.30, and 23.31) (McAdams et al. 1995). Tuberculous bronchiectasis may also occur in the lower lobes of the lung. It is usually asymptomatic, but secondary bacterial infection or hemoptysis may be a presenting feature.

\subsection{6}

\section{Pneumothorax as Complication of Post-primary Pulmonary TB}

Pneumothorax may occur during active post-primary pulmonary tuberculosis. Rupture of a tuberculous cavity contiguous to the pleura may result in pneumothorax. The incidence has been reported as low, ranging from $0.6 \%$ to $1 \%$ in hospitalized tuberculous patients (Wilder et al. 1962; Ihm et al. 1972). In our own series of 176 patients (see Table 20.2), pneumothorax occurred in $8(4.5 \%)$. In 1 patient, pneumothorax failed to respond to antituberculous treatment and chest tube insertion, and a bronchopleural fistula was suspected. A fistulogram confirmed the presence of a bronchopleural fistula and active tuberculosis in the apical segment of the left upper lobe (Fig. 23.36). Pneumothorax may also occur in treated and healed pulmonary tuberculosis (Lambert 1956). 


\subsection{7}

\section{ARDS and Tuberculosis}

Acute respiratory distress may occur in patients with bilateral chronic cavitary or bronchogenic pulmonary tuberculosis with a high hospital mortality rate of up to $47 \%$. Dyer and Potgieter (1984) described three adult patients from South Africa with adult respiratory distress syndrome (ARDS) due to pulmonary tuberculosis (nonmiliary).

A 31-year-old woman with cough, fever, and dyspnea lasting 2 months had bilateral pulmonary tuberculosis, and because of the severe dyspnea and the abnormal arterial blood gases, she was mechanically ventilated. The diagnosis of tuberculosis was made by sputum-positive direct smear. Antituberculous treatment was started, but the patient died. Postmortem examination showed left upper and right lower lobe cavitary tuberculosis. The second patient was a 22-year-old woman with a similar presentation who died in the ICU with postmortem evidence of bilateral tuberculous bronchopneumonia with a left bronchopleural fistula. The third patient had a similar presentation but did not require ventilation and responded well to antituberculous treatment.

Levy and colleagues (1987) from South Africa reported a retrospective study of 15 patients admitted to the ICU between January 1982 and June 1985 with a confirmed diagnosis of pulmonary tuberculosis. These patients required ICU admission for respiratory failure. They comprised $1.5 \%$ of 933 patients with pulmonary tuberculosis hospitalized in Hillbow Hospital. Eleven patients required ventilation, and 5 died. Miliary tuberculosis was found in 6 patients, tuberculous bronchopneumonia in 5 patients, lobar pneumonia in 3 patients, bilateral lung parenchymal destructive disease in 2.

Penner and colleagues (1995) from Canada reviewed the records of 13 patients with confirmed pulmonary tuberculosis ( 7 women and 6 men) from 1984 to 1993, and all had respiratory failure requiring mechanical ventilation. Seven patients had miliary or disseminated tuberculosis and 6, tuberculous pneumonia. Nine patients died, and only 4 survived. M. tuberculosis was isolated in all patients from respiratory or nonrespiratory sites. These authors also quoted the work of Agarwal and colleagues (1977) regarding 16 patients with respiratory failure due to tuberculous pneumonia who required mechanical ventilation.

\subsection{8}

\section{Tuberculous Bronchopleural Fistula}

Tuberculous bronchopleural fistula is rarely reported nowadays with the recent advances in chemotherapy. Most recent reports are on patients with late complications of collapse therapy for pulmonary tuberculosis that was done in the past (Johnson et al. 1973; Iseman and Madsen 1991; Uchida et al. 1999; Weissberg and Weissberg 2001). It may develop after lung resection for pulmonary tuberculosis. The lung parenchymal tuberculous cavity may rupture into the pleural space with pneumothorax, and the diagnosis can be made by fistulography (Fig. 23.36 radiology of pulmonary TB chapter). A tract may form between the bronchus contiguous to the tuberculous cavity and the pleura, producing a bronchopleural fistula. It is estimated that a bronchopleural fistula can occur in patients with active extensive pulmonary parenchymal tuberculosis in less than 1\% (Miller 1981; Woodring 1986; WinerMuram and Rubin 1990). Patients are usually symptomatic, and sputum production may increase. Plain chest radiography may show air in the pleural space, a changing air-fluid level, and contralateral spread of tuberculous infiltration. CT may depict the sites of the bronchopleural fistula (Kim et al. 2001).

The management of these patients is usually difficult. Patients with a susceptible organism may respond to antituberculous treatment and intercostal tube drainage. Patients with multidrug-resistant tuberculosis are treated with chemotherapy and lobectomy or pneumonectomy and pleural decortication unless found to have respiratory insufficiency (Iseman and Madsen 1991). Endobronchial occlusion by coils has been found to be an effective method (Uchida et al. 1999).

\subsection{9 \\ Aspergilloma (Mycetoma)}

A chronic tuberculous cavity may be colonized by the spores of Aspergillus fumigatus to form a fungal ball (Aderaye and Jajaw 1996). It may occur in $11 \%$ of patients with a chronic tuberculous cavity, and approximately $25 \%-55 \%$ of patients with aspergilloma have a history of the disease (Kim et al. 2001). Patients with aspergilloma are often asymptomatic but may present with hemoptysis (Kaestel et al.1999). Plain radiography of the chest may depict a mobile, rounded mass with air-crescent ring (Fig.23.26b and 23.37a,b). Surgical resection is essential if associated with hemoptysis and systemic antifungal treatment is ineffective. 


\subsubsection{0}

\section{Tuberculomas}

Solitary or multiple, round or oval, tuberculous pulmonary mass lesions may be noted on the chest radiographs in approximately $5 \%$ of patients (Kim et al. 2001; Winer-Muram and Rubin 1990). They may be the only radiographic manifestation of primary or post-primary pulmonary tuberculosis. Their diameter may range between 0.5 and $4.0 \mathrm{~cm}$ or larger with a smooth or sharply defined margin. Central caseation or calcifications may be found in $20 \%-30 \%$ of tuberculomas. Satellite lesions may be seen in up to $80 \%$ of these lesions.

\subsubsection{1}

\section{Tuberculous Pulmonary Gangrene}

An extremely rare but fatal complication of tuberculous cavitary lesion is the involvement of adjacent vessels and the development of arterial and venous vasculitis with thrombosis, leading to pulmonary gangrene (Reich 1993). Khan and colleagues (1980) from New York reported on 4 patients with pulmonary gangrene due to pulmonary tuberculosis, and only 1 survived. The first patient was a 55-year-old man who presented with fever, cough, and hemoptysis lasting 3 months. Chest radiography showed bilateral upper lobe infiltrate with cavitation and intracavitary mass. The sputum smear was positive for the bacilli. Chemotherapy was started, but the patient developed pneumothorax and died. Autopsy showed tuberculous pneumonia with extensive tuberculous arteritis and occlusion of the lumen by thrombosis.

The second patient died before establishing the diagnosis, and subsequent sputum culture taken on admission grew M. tuberculosis. Autopsy showed pulmonary vasculitis in arteries and veins contiguous with the tuberculous cavity. The third patient was known to be tuberculous before and had undergone lobectomy, and now presented with fever and a large pulmonary cavity with an intracavitary mass in the right upper lobe. The sputum smear was positive, and culture grew $M$. tuberculosis. She responded well to chemotherapy. The fourth patient had a similar chest radiographic cavity with intracavitary mass and died. The bacilli were found at autopsy as well as lung parenchymal cavities, tuberculous granulomatous vasculitis with thrombosis of the pulmonary arteries and veins.

We reviewed the world literature and found 18 previously reported patients with pulmonary gangrene mostly due to Klebsiella pneumonia and other organisms but not due to M. tuberculosis. Lopez-Contreras et al. (1994) reported a 61-year-old alcoholic man with a 4-month history of cough, fever, and weight loss. Sputum smear and culture were positive for M. tuberculosis. Chest radiography showed a large cavity with air-fluid level and a free-floating mass. The patient died, and autopsy was denied.

\subsubsection{2}

\section{Tuberculosis, Lung Cancer and Other Neoplasia}

The relationship between pulmonary tuberculous and lung cancer has been frequently debated. Such a relationship was raised by Greenberg and colleagues (1964), indicating the co-existence of carcinoma and tuberculosis of the lung. The co-existence between bronchogenic carcinoma and tuberculosis creates a difficult diagnostic problem for radiologists as the radiographic changes may be misinterpreted as progression of tuberculosis (Kim et al. 2001; WinerMuram and Rubin 1990).

Brown and Almenoff (1992) reviewed the literature of various retrospective studies on this co-existence. They reviewed other malignancies including leukemia, lymphoma, myelofibrosis, head and neck malignancies, as well as the use of immunosuppressive chemotherapy, and their relationship with the development of tuberculosis. They noted that tuberculosis was 6-9 times more common among patients with Hodgkin's disease, lung cancer, and non-Hodgkin's lymphoma than those with other malignancies. These authors noted, 'Tuberculosis was more likely to be diagnosed at the time of tumor diagnosis in patients with lung, head and neck malignancies, and disease in these patients was predominantly confined to the lungs'. Profound suppression of the cell-mediated immune response caused by malignant diseases or as a result of severe immunosuppressive chemotherapy is the most likely cause of the high risk of developing tuberculosis among these patients (Brown and Almenoff 1992).

\section{3 \\ Diagnosis of Post-primary Pulmonary TB}

20.3.1

Imaging Features of Post-primary Pulmonary TB

Various imaging modalities are used for the depiction of features of post-primary pulmonary 
tuberculosis. The imaging features of post-primary pulmonary tuberculosis can be broadly classified as lung parenchymal disease with cavitation, endobronchial tuberculosis, pleural extension of the disease, and other complications such as tuberculoma and mycetoma.

Conventional plain chest radiography is the mainstay imaging modality in depicting pulmonary features of the disease. However, a normal chest radiograph does not exclude pulmonary tuberculosis and has been reported in approximately $10 \%-20 \%$ of immunocompetent and immunocompromised patients, respectively (Fitzgerald et al. 1991; Miller and Miller 1993; Greenberg et al. 1994; Lee and Im 1995).

Computed tomography (CT) is useful in depicting cavitation and in patients with pleural effusion that may be masking lung parenchymal involvement on the same side (Kuhlman et al. 1990).CT is more sensitive than chest radiography (Hulnick et al. 1983; Lee et al.1996) in depicting mediastinal and paratracheal lymphadenopathy, endobronchial tuberculosis, and dissemination to the lung parenchyma and other rare complications (Hatipoglu et al. 1996).

Bronchography, now replaced by CT, is still used as the investigation of choice for the detection of bronchiectasis in poor-recourse countries of endemic areas (Fig. 23.29). Arteriography for diagnostic or therapeutic methods of bronchial artery embolization (BAE) is used for these rare but life-threatening massive hemoptysis cases.

Imaging features of post-primary pulmonary tuberculosis, although suggestive, are not characteristic as they can simulate other diseases (Lee and Im 1995). Upper lobe infiltration or consolidation should always be suspected as tuberculous. Cavitation may occur in approximately $40 \%$, and the diagnosis of tuberculosis is usually not difficult if it is present in the upper lobe and associated with bronchogenic spread to other parts of the lung (Miller and Miller 1993). In some instances, upper lobe consolidation should always be considered to be tuberculosis until proven otherwise. The presence of lung parenchymal scarring, fibronodular or calcific changes should not be assumed as inactive, and follow-up is essential. We retrospectively reviewed the radiological records of 176 adult patients with post-primary pulmonary tuberculosis who had positive sputum culture for $M$. tuberculosis. They attended our hospital between 1998 and 2000, and their imaging features and frequencies are presented in Table 20.2.

The imaging features of post-primary pulmonary tuberculosis have been reported by us in a separate chapter (Chap. 23).

\subsection{2 \\ Microbiology of the Sputum and Bronchial Aspirate}

Sputum microscopy for the detection of acid-fast bacilli by the Ziehl-Nielsen stain remains the cornerstone of rapid diagnosis of pulmonary tuberculosis (Maartens 2002). In HIV patient's sputum, microscopy is positive less often. At least 3 single specimens of sputum should be initially collected from patients with productive cough. In those who have difficulty in providing sputum, an aerosol inhalation of sterile hypertonic saline can be used to stimulate sputum production. Morning gastric aspiration, bronchoalveolar lavage, or transbronchoscopic brush or biopsies may be required.

At least 5,000 to 10,000 bacilli per milliliter of sputum must be present to enable the detection of the organism by stained smear (Hobby et al. 1973). Sputum culture will require 10 to 100 bacilli to yield a positive result (Yeager et al. 1967).

The American Thoracic Society (2000) reported on the diagnostic standards and classifications of tuberculosis in adult and children, and indicated that $50 \%-80 \%$ of patients with pulmonary tuberculosis will have positive sputum smears. Traditional culture media required 4-8 weeks to yield the bacilli, while radiometric culture methods (BACTEC) combined with a DNA probe allow identification of $M$. tuberculosis in 1-3 weeks.

Currently, PCR-based assays for the diagnosis of tuberculosis and identification of drug-resistant strains are configured to yield results in a few hours to days (see Chapter PCR and Diagnosis of Tuberculosis by Dr. Diana L. Williams).

\subsection{3 \\ Bronchoscopic Diagnosis}

The use of bronchoscopy to obtain diagnostic specimens for patients with a chest radiograph suggesting tuberculosis but with negative sputum specimen has been reported (Willcox et al. 1982). Willcox and colleagues reported on 275 patients seen from 1976 to 1980 , with imaging features of suspected tuberculosis and negative sputum smear. Specimens collected by bronchoscopy included bronchial brushings in 83, transbronchial lung biopsies in 18 , and post-bronchoscopy sputum. Positive brushing yield was $67.5 \%$, and transbronchial biopsies were positive in $50 \%$. They also reported the co-existence of tuberculosis and bronchial carcinoma in 4 of their patients (4\%). 
In our series of 176 patients, bronchoscopy was done on 27 patients with a chest radiograph suggesting pulmonary tuberculosis but with negative direct smear. Tuberculosis was confirmed by positive culture of bronchoscopic specimens, and no bronchial carcinoma was found.

Tuberculous infection after bronchoscopy may be transmitted from patient to patient. Molecular epidemiological studies by molecular typing of DNA supported the transmission of $M$. tuberculosis isolates to other patients via bronchoscopic contamination (Michele et al. 1997; Argeton et al. 1997). It is recommended that the instrument be cleaned prior to its immersion in $2 \%$ aqueous solutions of glutaraldehyde for 45-min exposure times (Food and Drug Administration 1992).

\subsection{4}

\section{Tuberculin Skin Test}

The tuberculin skin test is still widely used to identify infection with $M$. tuberculosis. Antigenic extracts of culture 'PPD' produces a delayed-type hypersensitivity reaction. Intradermal injection of $0.1 \mathrm{mg} / 0.1 \mathrm{ml}$ of the standard 5-tuberculin unit (TU) dose (Mantoux method) is done into the volar or dorsal surface of the forearm (American Thoracic Society 2000). The test should be read between 48 and $72 \mathrm{~h}$ after injection. Three cut-off points have been recommended for defining a positive PPD test. A cut-off point of $\geq 5 \mathrm{~mm}$ of induration using the ballpoint pen method of Sokal is considered positive in a person with recent contact or in the presence of abnormal chest radiographs consistent with tuberculosis. A cut-off point of $\geq 10 \mathrm{~mm}$ is suggested for individuals who have normal or mildly impaired immunity and a high likelihood of being infected with the disease but without other risk factors. A cut-off point of $\geq 15 \mathrm{~mm}$ for individuals with no risk factors for tuberculosis is considered positive (American Thoracic Society 2000). Tuberculin tests have several limitations including difficult administration, anergy, poor specificity, and the need for repeated testing to detect boosting (Maartens 2002). A comparative study between skin tests with PPD and measurement of the response by in-vitro culture assays measuring IFN- $\gamma$ production in response to tuberculin antigen stimulation is described in detail in a separate chapter. Dr. Rohit Katial has demonstrated the superiority of these assays and compared it with the PPD skin test (see Chap. 15).

\subsection{5 \\ Other Diagnostic Investigations}

Ultrasound or CT-guided, transthoracic, percutaneous, fine-needle aspiration (FNA) cytology may be useful in diagnosing pulmonary tuberculosis in patients initially suspected of having malignancies. Das et al. (1995) reported the use of FNA to diagnose tuberculosis in 29 patients in their series of 190 patients with malignancies and other causes.

Serological tests for antimycobacterial antibodies in the serum using an ELISA immunoassay may be positive in up to $88 \%$ (Barnes et al. 1993). DNA fingerprinting PCR-based assays provide a noninvasive method of diagnosing M. tuberculosis as well as identifying drug-resistant strains, with results ready in a few hours or days (see Chap. 13).

Pulmonary function tests may be required in tuberculous patients, particularly when lung surgery is required. Radionuclide studies in pulmonary tuberculosis are useful imaging tools in assessment of the disease (see Chap. 24 and 26).

\section{4 \\ Treatment of Post-primary Pulmonary TB}

The history of the management of tuberculosis has been called 'the story of medical failure' (Holme 1998). 'The patients have been blamed for non-compliance with the therapeutic regimen, but sociologic studies have shown that, in most cases, the providers of health care are at fault' (Grange and Zumla 2000). The infectivity of tuberculous patients for close contacts after starting chemotherapy has been studied by several authors. Riley et al. (1962) reported that the effluent air from the rooms of patients receiving chemotherapy became noninfectious for guinea-pigs within 2 weeks. Other authors found the rapid reduction in the number of viable bacilli by $1-2$ logarithmic counts within 2 weeks (Yeager et al. 1967; Hobby et al. 1973; Jindani et al. 1980). An editorial (1980) reviewed the issue of isolation of infectious patients with pulmonary tuberculosis, indicating that admission to a sanatorium was justified for supervised treatment, considering its toxicity and to safeguard their close contacts. It also referred to the work of Jenkinson et al. (1979) who found viable bacilli in the sputum of 15 patients who had received 6 weeks of treatment, and active disease was produced when injected into guinea-pigs. Studies on the duration of antituberculous treatment for patients with a positive 
sputum smear to achieve three consecutive negative results has been carefully studied prospectively by Telzak and colleagues (1997) from the South Bronx in New York. The study started from April 1993 to March 1995 of all patients with culture-confirmed tuberculosis. Data included the results of smears, cultures, and drug susceptibility, HIV status, CD4 cell count (for HIV-positive patients). The main objective of the study was to identify the time duration between initiating antituberculous treatment to the first of three consecutive negative sputum smears and the first of three consecutive negative sputum cultures. During the period of the study, 199 patients with culture-positive tuberculosis were diagnosed, $75 \%$ had lung parenchymal disease alone, $2 \%$ had lung and pleural abscess, $14 \%$ had pulmonary and extrapulmonary $\mathrm{TB}, 3 \%$ had only pleural disease, and $7 \%$ had extrapulmonary disease alone. They had complete information on 100 of the sputum smearpositive patients $(85 \%)$. They found that the mean number of days before the first of three consecutive negative sputum smears was 33 , and the median was 23 days. The mean number of days until the first of three consecutive negative sputum cultures was 32 , and the median was 26 days. These authors noted that the following factors were associated with an increased number of days to achieve their objective: the high number of AFB on initial smear, the presence of cavitary disease, and no previous history of tuberculosis. They noted that HIV had no effect on the duration of treatment before achieving the study objectives. HIV-positive patients are less likely to have cavitary disease, and therefore more likely to produce few AFB. In conclusion, patients with smearpositive sputum require hospitalization and must be isolated for a mean duration of 32 days after initiating appropriate treatment or longer if the initial AFB count was high in the presence of cavitary disease and if the patient had no prior history of tuberculosis.

The aim of the appropriate chemotherapy is to use drugs to which the bacilli are susceptible, with bactericidal activity to cure the patient, reduce infectivity to contacts, and prevent the emergence of drug resistance (Centers for Disease Control 1994). Clinicians are often required to take the decision of either to initiate the treatment of smear-negative patients with a presumptive diagnosis of pulmonary tuberculosis based on the presence of clinical and imaging features, or to wait for the sputum culture results that may require 4-8 weeks to obtain and may not yield the bacilli. In a prospective study of 139 patients with a presumptive diagnosis of pulmonary tuberculosis, Gordin et al. (1989) reported their findings. The study was carried out in San Francisco between mid1981 to the end of 1982 . The presumptive diagnosis in this series was based on the presence of clinical and radiological features suggestive of pulmonary tuberculosis with negative sputum smear. Treatment with isoniazid, rifampicin, and ethambutol was given to all patients. Positive culture was reported later in 16 patients. Among the culture-negative patients, 43 showed radiographic improvement after 3 months of initiating the treatment, clinical improvement in 7 , and bronchoscopic confirmation of the diagnosis in 1 patient.

There were 72 individuals with radiological stability who were considered as having inactive previous tuberculosis. The authors concluded that the treatment was appropriate in 66 of 139 (48\%) of patients. The British Medical Research Council (BMRC) recommended the initiation of antituberculous treatment for such patients after other causes for abnormal chest radiography findings have been excluded (Dutt and Stead 1994; Fox et al. 1999).

The modern short course of antituberculous chemotherapy, Directly Observed Therapy (DOT), is the best strategy for the treatment of post-primary pulmonary tuberculosis to achieve the three goals, that is, cure the patient, reduce infectivity to contacts, and prevent the emergence of drug resistance if appropriately adhered to. DOT is usually given as an outpatient treatment and supervised by nurses. Such visits to the outpatient facility may be difficult as it involves traveling and may be costly for patients in developing countries. Training of a family member or a community lay person to supervise the administration of DOT may be more practical in developing countries (Wilkinson 1994). WHO indicated that for the DOT strategy to be effective, it requires government commitment, the availability of microscopic and other diagnostic facilities, a continuous supply of high-quality medication, direct observation of treatment administration, and recording the response to treatment (Netto et al. 1999).

DOT consists of an initial 2-month phase of intensive treatment using four drugs (rifampicin, isoniazid, pyrazinamide, and either ethambutol or streptomycin) followed by a 4-month continuation phase of rifampicin and isoniazid. These drugs are usually given as daily treatment but can be given thrice weekly, either throughout or during the continuation phase, to facilitate the supervision of treatment. The isoniazid daily dose is $5 \mathrm{mg} / \mathrm{kg}$ orally or intramuscularly (maximum $300 \mathrm{mg}$ ), rifampicin daily dose is $10 \mathrm{mg} / \mathrm{kg}$ orally (maximum $600 \mathrm{mg}$ ), pyrazinamide daily dose is $15-30 \mathrm{mg} / \mathrm{kg}$ orally (maximum $2 \mathrm{~g}$ ), 
streptomycin daily dose is $15 \mathrm{mg} / \mathrm{kg}$ intramuscularly (maximum $1 \mathrm{~g}$ ) for persons below the age of 60 years and $10 \mathrm{mg} / \mathrm{kg}$ intramuscularly (maximum $750 \mathrm{mg}$ ) for persons above 60 years, ethambutol daily dose is $15-25 \mathrm{mg} / \mathrm{kg}$ orally (maximum $2-5 \mathrm{~g}$ ). The cure rate of drug-susceptible disease when the patient completes the course of treatment is up to $98 \%$. HIV co-infected patients have a similar outcome of tuberculosis treatment success rate (Grange and Zumla 2000). Although DOT has been successful in some countries, endemic areas with HIV and MDR tuberculosis have not achieved the WHO targets for disease control (Netto et al. 1999).

Multidrug-resistant tuberculosis is a much more serious problem to treat. Drugs used for treatment are more toxic, more expensive, and require a prolonged period of up to 24 months, and surgery may be considered to decrease the load of the organisms during chemotherapy (Iseman 1993; Iseman et al. 1990).

\subsection{1 \\ Response to Treatment}

Appropriate chemotherapy is usually associated with clinical and radiological improvements. Clinical improvement with resolution of fever occurred in $92 \%$ of patients 4 weeks after starting treatment (Teklu and Al-Wabel 1994; Vanham et al. 1997). Radiological improvement is noticed after 3 months (Gordin et al. 1989). Paradoxical worsening of the clinical and imaging responses to treatment may occur particularly in the first few weeks or months. HIV co-infected patients are more prone to paradoxical worsening after starting antituberculous treatment, and this is reported to occur in $36 \%$ (Wendel et al. 2001). In tuberculous patients without HIV, paradoxical worsening may occur in 16\% (Al-Majed 1996). Paradoxical worsening after antituberculous treatment is defined as worsening of fever, cough, shortness of breath or even development of ARDS, enlargement of lymph nodes or other extrapulmonary site of the disease during appropriate treatment (Smith 1987; Al-Majed 1996; Rodriguez-Bano et al. 1997; Hung and Chang 1999; Wendel et al. 2001).

Radiological worsening of the pre-existing pulmonary infiltrates, development of new lesions on the same or apposite lung, enlargement of hilar or paratracheal lymph nodes were noted in our series (Figs. 23.16a-c and 23.17a-c). The pathogenesis of paradoxical worsening is not yet clear. It is believed that it may be due to enhanced immune responses (immunological rebound) with increased proliferation of mononuclear cells and IFN- $\gamma$ production in response to M. tuberculosis antigens (Wendel et al. 2001). Corticosteroid therapy has been used to modify the intensity of symptoms (Rodriguez-Bano et al. 1997). There have been no controlled trials on the management of these paradoxical worsening responses.

\section{References}

Abal AT, Nair PC, Cherian J (2001) Haemoptysis: aetiology, evaluation and outcome - a prospective study in a thirdworld country. Respir Med 95:548-552

Aderaye G, Jajaw A (1996) Bilateral pulmonary aspergilloma: case report. East Afr Med J 73:487-488

Agarwal MK et al. (1977) Respiratory Failure in Pulmonary tuberculosis. Chest 72:605-609

Al-Hajjaj MS, Pandya L, Marie AA et al (1991) Pulmonary tuberculosis in Saudi Arabia: a retrospective study of 1566 patients. Ann Saudi Med 11:443-447

Alland D, Kalkut GE, Moss AR (1994) Transmission of tuberculosis in New York City. An analysis by DNA fingerprinting and conventional epidemiologic methods. $\mathrm{N}$ Engl J Med 33:1710-1716

Al-Majed SA (1996) Study of paradoxical response to chemotherapy in tuberculous pleural effusion. Respir Med 90:211-214

Al-Wabel-A, Teklu B, Buluk O et al (1995) The spectrum of pulmonary tuberculosis in the Asir region of Saudi Arabia. SMJ 16:105-107

Alzeer A, Mashlah A, Fakim N et al (1998) Tuberculosis is the commonest cause of pneumonia requiring hospitalization during Hajj (Pilgrimage to Makkah). J Infect 36:303-306

American Thoracic Society (2000) Diagnostic standards and classification of tuberculosis in adults and children. Am J Respir Crit Care 161:1376-1395

Argeton T, Valway S, Gore B et al (1997) Transmission of a highly drug-resistant strain (strain W1) of Mycobacterium tuberculosis: community outbreak and nosocomial transmission via a contaminated bronchoscope. JAMA 278:1073-1077

Balasubramanian V, Wiegeshaus EH, Taylor BT et al (1994) Pathogenesis of tuberculosis: pathway to apical localization. Tuberc Lung Dis 75:168-178

Bandera A, Gori A, Catozzi L et al (2001) Molecular epidemiology study of exogenous reinfection in an area with a low incidence of tuberculosis. J Clin Microbiol 39:2213-2218

Barnes PF, Barrows SA (1993) Tuberculosis in the 1990 s. Ann Intern Med 119:400-410

Barnes PF, Bloch AB, Davidson PT et al (1991) Tuberculosis in patients with human immunodeficiency virus infection. $\mathrm{N}$ Engl J Med 324:1644-1650

Bates JH (2001) Reinfection tuberculosis. How important is it? Am J Respir Crit Care Med 163:600-601

Brande PVD, Dockx S, Demedts VM (1998) Pulmonary tuberculosis in the adult in a low prevalence area: is the radiological presentation changing? Int J Tuberc Lung Dis 2:904-908 
Brown ST, Almenoff PL (1992) Pulmonary mycobacterial infections associated with neoplasia. Semin Respir Infect 7:104-113

Buckner CB, Walker CW (1990) Radiologic manifestation of adult tuberculosis. J Thorac Image 5:28-37

Caminero JA, Pena MJ, Campos-Herrero MI et al (2001) Exogenous reinfection with tuberculosis on a European island with a moderate incidence of disease. Am J Respir Crit Care 163:717-720

Cartier Y, Kavanagh PV, Johkoh T et al (1999) Bronchiectasis: accuracy of high-resolution CT in the differentiation of specific diseases. AJR Am J Roentgenol 173:47-52

Centers for Disease Control (1994) Guidelines for preventing the transmission of Mycobacterium tuberculosis in health-care facilities. MMWR Morb Mortal Wkly Rep 43:1-132

Choyke PL, Sostman HD, Curtis AM et al (1983) Adult-onset pulmonary tuberculosis. Radiology 148:357-362

Conlan AA, Hurwitz SS et al (1983) Massive hemoptysis. J Thorac Cardiovasc Surg 85:120-124

Corbett EL, Churchyard GJ, Clayton TC et al (2000) HIV infection and silicosis: the impact of two potent risk factors on the incidence of mycobacterial disease in South African miners. AIDS 14:2759-2768

Dahmash NS, Fayed DF, Chowdhury MNH, Arora SC (1995) Diagnostic challenge of tuberculosis of the elderly in hospital: experience at a university hospital in Saudi Arabia. J Infect 31:93-97

Dankner WM, Waecker NJ, Essey MA et al (1993) Mycobacterium bovis infections in San Diego: a clinico-epidemiological study of 73 patients and a historical review of a forgotten pathogen. Medicine 72:11-37

Dannenberg AM Jr (2001) Pathogenesis of pulmonary Mycobacterium bovis infection: basic principles established by the rabbit model. Tuberculosis 81:87-96

Das DK, Pant CS, Pant JN et al (1995) Transthoracic (percutaneous) fine needle aspiration cytology diagnosis of pulmonary tuberculosis. Tuberc Lung Dis 76:84-89

Dunlap NE, Bass J, Fujiwara P et al (2000) Diagnostic standards and classification of tuberculosis in adults and children. Am J Respir Crit Care Med 161:1376-1395

Dutt AK, Stead WW (1994) Smear-negative pulmonary tuberculosis. Semin Respir Infect 9:113-119

Dyer RA, Potgieter PD (1984) The adult respiratory distress syndrome and bronchogenic pulmonary tuberculosis. Thorax 39:383-387

Editorial (1980) Isolation of patients with pulmonary tuberculosis. Br Med J (5 April):962-963

Fitzgerald JM, Gizybouski S, Allen EA (1991) The impact of human immunodeficiency virus infection on tuberculosis and its control. Chest 100:191-200

Food and Drug Adminsitration, Infection Control Devices Branch, Division of General and Restorative Devices, Office of Device Evaluation (1992) Guidance on the content and format of premarket notification (510[k]) Submissions for Liquid Chemical Germicides. Rockville, Md: Food and Drug Administration, 31 Jan

Fox W, Ellard GA, Mitchison DA (1999) Studies on the treatment of tuberculosis undertaken by the British Medical Research Council Tuberculosis Units, 1946-1986, with relevant subsequent publications. Int J Tuberc Lung Dis 3 [Suppl 2]:S231-S279

García M, Vargas JA, Castejón R et al (2002) Flow-cytometric assessment of lymphocyte cytokine production in tuberculosis. Tuberculosis 82:37-41

Geppert EF, Leff A (1979) The pathogenesis of pulmonary and miliary tuberculosis. Arch Intern Med 139:1381-1383

Glynn JR, Shearer S, Kambashi B, Godfrey-Fausett P (2001) HIV-I and recurrence, relapse, and reinfection of tuberculosis after cure: a cohort in South African mineworkers. Lancet 358:1687-1693

Gordin FM, Slutkin G, Schecter G et al (1989) Presumptive diagnosis and treatment of pulmonary tuberculosis based on radiographic findings. Am Rev Respir Dis 139:1090-1093

Grange JM, Zumla A (2000) Advances in the management of tuberculosis: clinical trials and beyond. Curr Opin Pul Med 6:193-197

Grange JM, Daborn C, Cosivi O (1994) HIV-related tuberculosis due to Mycobacterium bovis. Eur Respir J 7:1564-1566

Greenberg SD, Jenkins DE, Bahar D (1964) Coexistence of carcinoma and tuberculosis of the lung. Am Rev Respir Dis 90:67-76

Greenberg SD, Frager D, Suster B et al (1994) Active pulmonary tuberculosis in patients with AIDS: spectrum of radiographic findings (including a normal appearance). Radiology 193:115-119

Hatipoglu ON, Osma E, Manisali M et al (1996) High resolution computed tomographic findings in pulmonary tuberculosis. Thorax 1996:397-402

Heyderman RS, Goyal M, Roberts P et al (1998) Pulmonary tuberculosis in Harare, Zimbabwe: analysis by spoligotyping. Thorax 53:346-350

Hirshberg B, Biran I, Glazer M et al (1997) Hemoptysis: etiology, evaluation, and outcome in a tertiary referral hospital. Chest 112:440-444

Hobby G, Holman A, Iseman M et al (1973) Enumeration of tubercle bacilli in sputum of patients with pulmonary tuberculosis. Antimicrob Agents Chemother 4:94-104

Holme C (1998) Tuberculosis: story of medical failure. Br Med J 317:1260

Hsiao EI, Kirsch CM, Kagawa FT et al (2001) Utility of fiberoptic bronchoscopy before bronchial artery embolization for massive hemoptysis. AJR Am J Roentgenol 177:861-867

Hulnick DH, Naidich DP, McCauley DL (1983) Pleural tuberculosis evaluated by computed tomography. Radiology 149 : 759-765

Hung SC, Chang SC (1999) New pulmonary lesions during therapy for extrapulmonary tuberculosis. Chest 116:1794-1797

Ihm HJ, Hankins JR et al (1972) Pneumothorax associated with pulmonary tuberculosis. J Thorac Cardiovasc Surg 64:211-219

Im JG, Itoh H, Shim YS (1993) Pulmonary tuberculosis: CT findings-early active disease and sequential change with antituberculous therapy. Radiology 186:653-660

Iseman MD (1993) Treatment of multidrug-resistant tuberculosis. N Engl J Med 329:784-791

Iseman MD, Madsen LA (1991) Chronic tuberculous empyema with bronchopleural fistula resulting in treatment failure and progressive drug resistance. Chest 100:124-127

Iseman MD, Madsen L, Goble M et al (1990) Surgical intervention in the treatment of pulmonary disease caused by drug-resistant Mycobacterium tuberculosis. Am Rev Respir Dis 141:623-625

Jindani A, Aber V, Edwards E et al (1980) The early bacterial activity of drugs in patients with pulmonary tuberculosis. Am Rev Respir Dis 121:939-949 
Johnson JL, Ellner JJ (2000) Adult tuberculosis overview: African versus Western perspectives. Curr Opin Pulm Med 6:180-186

Johnson TM, McCann et al (1973) Tuberculous bronchopleural fistula. Am Rev Respir Dis 107:30-41

Kaestel M, Meyer W, Mittelmeier HO et al (1999) Pulmonary aspergilloma - clinical findings and surgical treatment. Thorac Cardiovasc Surg 47:340-345

Khan FA, Rehman M et al (1980) Pulmonary gangrene occurring as a complication of pulmonary tuberculosis. Chest 77:76-80

Kim HY, Song KS et al (2001) Thoracic sequelae and complications of tuberculosis. Radiographics 21:839-860

Kuhlman JE et al (1990) CT features of thoracic mycobacterial disease. Radiographics 10:413-431

Lambert HP (1956) Spontaneous pneumothorax and pulmonary tuberculosis. Tubercle 37:207-209

Lee KS, Im JG (1995) CT in adult with tuberculosis of the chest: Characteristic findings and role of management. AJR 164: 1361-1367

Lee KS, Kim YH, Kim WS et al (1991) Endobronchial tuberculosis: CT features. J Comput Assist Tomogr 15:424-428

Lee KS, Hwang JW, Chung MP et al (1996) Utility of CT in the evaluation of pulmonary tuberculosis in patients without AIDS. Chest 110:977-984

Lee TW, Wan S, Choy DK et al (2000) Management of massive hemoptysis: a single institution experience. Ann Thorac Cardiovasc Surg 6:232-235

Levy H, Kallenbach JM, Feldman C et al (1987) Acute respiratory failure in active tuberculosis. Crit Care Med 15: 221-225

Liesegang TJ, Cameron Douglas (1980) Mycobacterium bovis infection of the conjunctiva. Arch Ophtalmol 98:1764-1766

Lopez-Contreras JL, Ris J et al (1994) Tuberculous pulmonary gangrene: report of a case and review. Clin Infect Dis 18: 243-245

Maartens G (2002) Advances in adult pulmonary tuberculosis. Curr Opin Pulm Med 8:172-177

Maartens G, Beyers N (2002) Tuberculosis in the tropics. Clin Chest Med 23:341-350

Mal H, Rullon I, Mellot F (1999) Immediate and long-term results of bronchial artery embolization for life-threatening hemoptysis. Chest 115:996-1001

McAdams HP, Erasmus J et al (1995) Radiologic manifestations of pulmonary tuberculosis. Radiol Clin North Am 33:655-678

McDonough KA, Florczyk MA, Kress Y (2000) Intracellular passage within macrophages affects the trafficking of virulent tubercle bacilli upon reinfection of other macrophages in a serum-dependent manner. Tuberc Lung Dis 80:259-271

Michele TM, Cronin WA, Graham NMH et al (1997) Transmission of Mycobacterium tuberculosis by a fiberoptic bronchoscope: identification by DNA fingerprinting. JAMA 278: 1093-1095

Millar JW, Horne NW (1979) Tuberculosis in immunosuppressed patients. Lancet 1:1176-1178

Miller WT (1981) Tuberculosis in the adult. Postgrad Radiol 1:147-167

Miller WT, Miller WT (1993) Tuberculosis in the normal host: radiological findings. Semin Roentgenol 28:109-118

Moda G, Daborn CJ, Grange JM et al (1996) The zoonotic importance of Mycobacterium bovis. Tuberc Lung Dis 77:103-108
Netto EM, Dye C, Raviglione MC (1999) Progress in global tuberculosis control 1995-1996, with emphasis on 22-highincidence countries. Global Monitoring and Surveillance Project. Int J Tuberc Lung Dis 3:310-320

Penner C, Roberts D, Kunimoto D et al (1995) Tuberculosis as a primary cause of respiratory failure requiring mechanical ventilation. Am J Respir Crit Care Med 151:867-872

Reich JM (1993) Pulmonary gangrene and the air crescent sign. Thorax 48:70-74

Riley R, Mills C, O'Grady F et al (1962) Infectiousness of air from a tuberculosis ward. Ultraviolet irradiation of infected air: comparative infectiousness of different patients. Am Rev Respir Dis 85:511-525

Rodriguez-Baño JR et al (1997) Systemic paradoxical response to antituberculous drugs: resolution with corticosteroid therapy. Clin Infect Dis 24:517-519

Rohenberg GT, Shaw P (1996) Radiology of pulmonary tuberculosis. Br J Hosp Med 56:195-199

Rook GAW, Zumla A (2001) Advances in the immunopathogenesis of pulmonary tuberculosis. Curr Opin Pulm Med 7:116-123

Sauret J, Jolis R, Ausina V et al (1992) Human tuberculosis due to Mycobacterium bovis: report of 10 cases. Tuberc Lung Dis 73:388-391

Small PM, Hopewell PC, Singh SP et al (1994) The epidemiology of tuberculosis in San Francisco. A population-based study using conventional and molecular methods. $\mathrm{N}$ Engl J Med 330:1703-1709

Smith H (1987) Paradoxical responses during the chemotherapy of tuberculosis. J Infect 15:1-3

Sokal JE (1975) Measurement of delayed skin-test responses. N Engl J Med 293:501-502

Stead WW, Bates JH (2000) Recurrent tuberculosis due to exogenous reinfection. N Engl J Med 341:1050

Stebbings AE, Lim TK (1999) Cause, treatment and outcome of patients with life-threatening haemoptysis. Singapore Med J 40:67-69

Teklu B, Al-Wabel AH (1994) Resolution of fever in patients on chemotherapy for pulmonary tuberculosis. Ann Saudi Med 14:392-395

Telzak EE, Fazal BA, Pollard CL et al (1997) Factors influencing time to sputum conversion among patients with smearpositive tuberculosis. Clin Infect Dis 25:666-670

Uchida T, Wada M, Sakamoto J et al (1999) Treatment for empyema with bronchopleural fistulas using endobronchial occlusion coils: report of a case. Jpn J Surg 29:186-189

Van Rie A, Warren R, Richardson M et al (1999) Exogenous reinfection as a cause of recurrent tuberculosis after curative treatment. $\mathrm{N}$ Engl J Med 341:1174-1179

Vanham G, Toossi Z, Hirsch CS et al (1997) Examining a paradox in the pathogenesis of human pulmonary tuberculosis: immune activation and suppression/anergy. Tuberc Lung Dis 78:145-158

Wallis RS, Johnson JL (2001) Adult tuberculosis in the 21st century: pathogenesis, clinical features, and management. Curr Opin Pulm Med 7:124-133

Weissberg D, Weissberg D (2001) Late complications of collapse therapy for pulmonary tuberculosis. Chest 120:847-851

Wendel KA, Alwood KS, Gachuhi R et al (2001) Paradoxical worsening of tuberculosis in HIV-infected persons. Chest 120:193-197

Wilder RJ, Beacham EG et al (1962) Spontaneous pneumothorax complicating cabitary tuberculosis. J Thorac Cardiovasc Surg 43:561-573 
Wilkinson D (1994) High-compliance tuberculosis treatment program in a rural community. Lancet 343:647-648

Willcox PA, Benatar SR, Potgieter (1982) Use of the flexible fibreoptic bronchoscope in diagnosis of sputum-negative pulmonary tuberculosis. Thorax 37:598-601

Winer-Muram HT, Rubin SA (1990) Thoracic complications of tuberculosis. J Thorac Imag 5:46-63
Wong ML, Szkup P, Hopley MJ (2002) Percutaneous embolotherapy for life-threatening hemoptysis. Chest 121:95-102

Woodring JH (1986) Update: the radiographic features of pulmonary tuberculosis. AJR 146:497-506

Yeager HJ, Lacy J, Smith L et al (1967) Quantitative studies on mycobacterial populations in sputum and saliva. Am Rev Respir Dis 95:998-1004 